\title{
Development of Trees Management System Using Radial Basis Function Neural Network for Rain Forecast
}

\author{
Hasnul Auzani*, Khairusy Syakirin Has-Yun, Farah Aniza Mohd Nazri \\ International Institute for Halal Research and Training, International Islamic University Malaysia, Selangor, Malaysia \\ Email: `hasnul.hassan@gmail.com, syakirin@iium.edu.my, anizafarah@gmail.com
}

How to cite this paper: Auzani, H., Has-Yun, K.S. and Nazri, F.A.M. (2022) Development of Trees Management System Using Radial Basis Function Neural Network for Rain Forecast. Computational Water, Energy, and Environmental Engineering, 11, 1-10.

https://doi.org/10.4236/cweee.2022.111001

Received: January 4, 2021

Accepted: November 28, 2021

Published: December 1, 2021

Copyright $\odot 2022$ by author(s) and Scientific Research Publishing Inc. This work is licensed under the Creative Commons Attribution International License (CC BY 4.0).

http://creativecommons.org/licenses/by/4.0/

(c) (i) Open Access

\begin{abstract}
Agriculture and farming are mainly dependent on weather especially in Malaysia as it received heavy rainfall throughout the years. An efficient crop or tree management system with a weather forecast needed for suitable planning of farming operation. Radial Basis Function Neural Network (RBFNN) algorithm was used in this study to predict rainfall and the main focus of this study is to analyze the factor that affects the performance of neural model. This study found that the model works better the more hidden nodes and the optimum learning rate is 0.01 with the RMSE $49 \%$ and the percentage accuracy is $57 \%$. Besides that, it is found that the meteorology data also affect the model performance. Future research can be conducted to improve the rainfall forecast of this study and improve the tree management system.
\end{abstract}

\section{Keywords}

Tree Management, Radial Basis Function, Rain Prediction, Artificial Neural Network

\section{Introduction}

Trees generally play a critical role across numerous significant aspects in human's livelihood. In the metropolitan areas, trees can be advantageous in creating an environment that is highly systematic as they contribute to the large production of oxygen as well as the reduction of gaseous pollutants, hence improving the general air quality. However, it is also important to note that, tree planting in the cities requires excellent planning which includes the execution of a well-planned and highly efficient tree management system by the local municipalities. Successful tree growth depends on the ability to manage such factors as 
pests, diseases, water, fertilizer requirements, etc. Even though the data stemming from the monitoring of tree management activities could only be tracked once trees have been planted but there are some studies which have demonstrated that the data stemming would affected by the tree development [1]. Nevertheless, this is not an overnight process and intensive sources with higher number of datasets are frequently difficult to find and access.

Quantitative forecasting for weather element such as rainfall, humidity, temperature, and others is valuable in agricultural areas [2]. This is because, factoring in the weather element in the tree-management system or planning could substantially drive down the operational costs associated to it. Better scheduling of activities pertaining to the management of trees planted can also be derived if weather forecast is being taken into consideration as an important part of the planning process. This, therefore, has demonstrated that there is a need to innovate the existing system as to incorporate the weather element due to Malaysia's climatic conditions.

Weather forecast is not only crucial in the urban planning to mitigate the risks stemming from weather that include floods and landslides but also in the general management of trees at the local recreational facilities and parks. Weather forecasting is a challenging activity. The challenging nature of weather forecasting can be derived from the complex atmospheric processes that are usually not presented via a perfectly linear correlation. Comparing the traditional and numerical methods, ANN is found to be the best approach for the weather prediction [3]. According to the research by [4] that compares linear and non-linear technique for rainfall prediction, ANN models are having higher correlations compared to the linear models, indicating better generalization capacity of the non-linear models in predicting by producing lower error and fitting better with the measured data.

Weather forecasting can be done by leveraging on the Numerical Weather Model (NWP) a statistical and Machine Learning-based model [4]. This statement being proved by the studies in [5], shows that the machine learning based model give a better prediction than the linear regression algorithm to predict rainfall. An artificial neural network computational tool is strong and data driven. Its characteristic is self-adaptive, flexible which has the capacity to learn and handle nonlinear and tough underlying characteristics of any physical process with high grade of accuracy [2]. Thanks to its ability in providing a better solution to complex problems that significantly reduce noise and optimize approximations, ANN is getting more attention from a lot of scholars in their attempts to unravel the inner workings of weather forecasting process [6]. Traditional methodologies are often found to encounter constant challenges in addressing non-linear physical, spatial and temporal processes as well as the underlying uncertainties that exist in certain parameters used. The attributes of the ANN model have therefore made it clear about its suitability to be deployed in studies pertaining to weather forecasting. 
ANN consists of several techniques such as the feedforward neural networks, backpropagation neural networks and radial basis function neural network (RBFNN). RBFNN structure is similar to typical ANN structure, that consists of one hidden layer that contain three main parameter that is output weights, widths and centered [7]. [8] highlights that RBFNN is the most suitable machine learning technique to be deployed in the study that scrutinizes rainfall forecast in comparison to other techniques including Generic Programming, Support Vector Regression, M5-Rules, M5-Model trees and k-Nearest Neighbor. This argument has been further resonated by [9], in which they conclude that RBFNN provides higher accuracy of results in rainfall forecast as compared to the backpropagation neural network technique. Besides, another study conducted by [10] finds out that the use of multilayer perceptron is suitable for in Atner while RBFNN in Dharni RBFNN.

Thus, we have proposed a model of weather forecasting using Radial Basis Function Neural Networks (ANN) order to predict weather in a very effective and efficient way and to enhance the rainfall prediction in Malaysia by utilizing the RBFNN model. Other than its proven ability to provide a better rainfall prediction, this model also employs a better algorithm that is far more simplistic and with a faster learning capability. Rainfall prediction relies heavily on important weather-related parameters that include air pressure, temperature and wind speed that should certainly be considered in the development of a sound algorithm in rainfall prediction [11]. Therefore, an analysis has been conducted across a few meteorology datasets as data input centered around the important parameters such as temperature, windspeed, humidity as well as air pressure.

\section{Data and Research Methodology}

\subsection{Rainfall Data}

Having located near the earth's equator, Malaysia is one of the countries that is blessed with a tropical climate which implies that it is hot and humid throughout the year. The location chosen for this study is Klang, Selangor and the daily rainfall data for the trend analysis used for this study is sourced directly from the Malaysia's Department of Irrigation and Drainage with data ranging over the period of 6 years (from 1 January 2015 until 31 December 2020) recorded using the $400 \mathrm{~cm}^{2}$ Casella rain gauge. Three meteorological data parameters have been incorporated to enhance this study; temperature $\left({ }^{\circ} \mathrm{C}\right)$, humidity $(\%)$, air pressure (mbar) dan wind speed (km/hour). For this study specifically, $70 \%$ of the daily rainfall data collected is used for training data, while $15 \%$ for validation data and the last $15 \%$ rainfall data are used during testing process.

\subsection{Model Performance Technique}

The performance of a rainfall forecasting system is analyzed via two statistical analyses which are Root Mean Square Error (RMSE) as well as accuracy percentage. These statistical methods outline a comparison between the system rainfall 
prediction values with the measured rainfall data value. A lower error value indicates a better performance of rainfall forecast system. $(\varepsilon)$ represents the error percentage. The accuracy percentage and the Root Mean Square Error (RMSE) are calculated via the following formula:

$$
\begin{gathered}
\varepsilon(P)=\frac{R_{\text {predicted }}(P)-R_{\text {measured }}(P)}{R_{\text {measured }}(P)} \times 100 \\
\text { Percentage of accuracy }=1-\varepsilon(P) \\
\text { RMSE }=\sqrt{\frac{1}{N} \sum_{i=1}^{N}\left(R_{\text {prediction }}-R_{\text {measured }}\right)^{2}}
\end{gathered}
$$

\subsection{Radial Basis Function Neural Network}

The concept of Artificial Neural Network is a network composed by a collection of processing units that are computer-programmed based on the working of human brain [12]. The human brain is a complex system capable of processing a large amount of information at a time. The neural network is a processor that is made up of artificial neurons as the main the processing element [13]. The application of the neural network has been extensive in the areas of pattern classification, grouping, prediction and optimization among many others. Rainfall is one of the natural phenomena with non-linear attributes, thus, requiring a non-statistical method that is far more complex as to understand the rainfall behavioral patterns just like how the Artificial Neural Network does [13].

RBFNN was first introduced by Broomhead dan Lowe in the year 1988 [14]. This paper use the algorithm of Radial Basis Function Network (RBFN) because RBFN is one of the neural networks with capability to predict non-linear attributes and with faster learning speed. RBFN is also a particularly distinctive artificial neural network as it leverages on the basis function network as the active function. The application of RBFN is used widely particularly for function approximation, time series forecasting and classification. RBFN model that comprises of three layers, the input layer, the hidden layer and the output layer is shown in Figure 1 [15].

Every layer is made up of nodes that connected one another as illustrated by Figure 1. As demonstrated in Figure 1, every neuron is also connected with one another. This information will later enter the hidden layer where the learning process shall commence. This directly implies that the calculations are performed so that system learns the patterns by manipulating the weighted value until it matches the output value. Radial basis function is a function only depends on value of distance from the origin. Thus, the limitation of radial basis function formula must contain only real values. The transformation associated to every node located within the hidden layer using the gaussian function which is derived by the following formula:

$$
z_{j}(x)=\exp \left(-\frac{d_{j}^{2}}{d_{\sigma}^{2}}\right)
$$




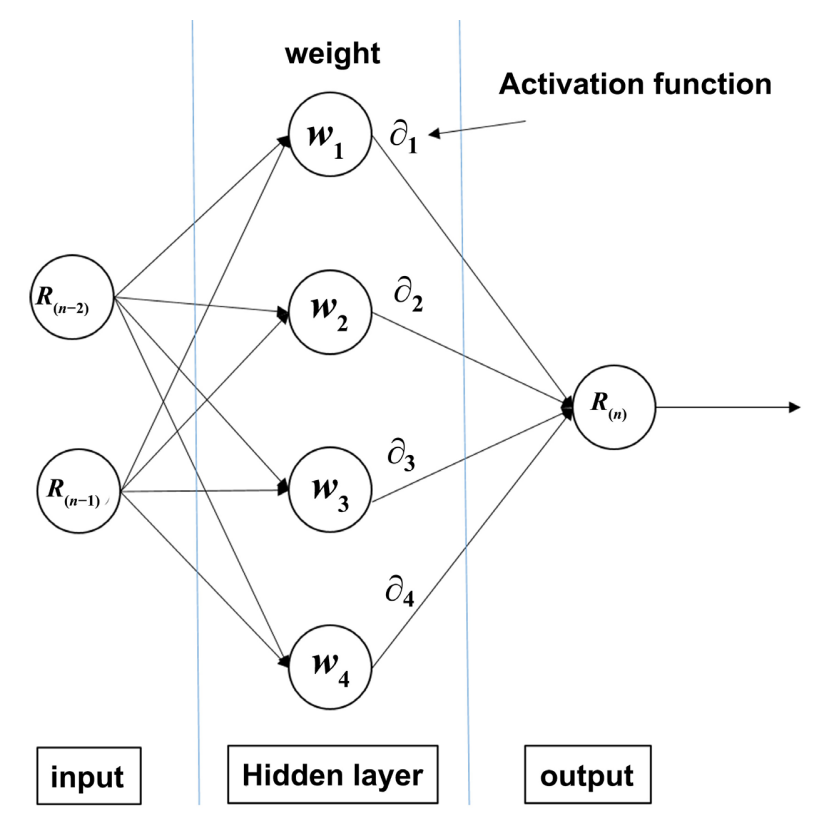

Figure 1. Radial basis function network (RBFN) modelling.

$$
d_{j}=\left|x-\mu_{j}\right|
$$

where $z_{j}(x)$ is the output from the hidden $j$ node and Bias node. $z_{0}(x)$ has a fixed value which is $1 . x$ is an input with n-dimension. $\sigma_{j}$ is the width of receptive field for the hidden $j$ node, $\mu_{j}$ is the centre of the hidden $j$ node and $d_{j}$ is the Euclidean distance between $x$ and $\mu_{j}$. On the output, all nodes are connected with one another. The output value, $y(x)$ RBFN is:

$$
y_{l}(x)=\sum_{j=0}^{j} w_{j l} z_{j}
$$

where $y_{l}$ is output node $l$ and $w_{j l}$ is the weight that has a relationship with the hidden $\mathrm{j}$ node and the output 1 node.

\section{Results and Discussion}

In this study, statistical analysis that has been used is root mean square error (RMSE) and the percentage of accuracy. The optimal RBFNN structure is determined by manipulating the hidden neuron value between 4 and 99 . The RMSE values between the predicted values and measured values for the RNFNN model are illustrated in Table 1 . This study discovers that the hidden neuron value has less affect on the performance of the RMSE value which is between $49.92 \%$ up to $51.10 \%$. However, in term of the percentage of accuracy, the hidden node value plays a significant role, in which, it leads to higher percentage of accuracy from the hidden nodes between $61 \%$ up to $99 \%$. This, therefore, has demonstrated that more hidden nodes will contribute to a better analysis. Nevertheless, it is essential to note that the increase in the number of nodes will add another layer of complexity for a neural network model. Hence, optimum selection of hidden nodes is one of the most important steps that should be taken during a neural network modelling process. 
Table 1. The preliminary performance of radial basis function neural network for hidden nodes.

\begin{tabular}{ccc}
\hline Number of hidden nodes & RMSE & Percentage of accuracy (\%) \\
\hline $4-4-1$ & 50.23 & 43.25 \\
$4-7-1$ & 50.23 & 43.25 \\
$4-8-1$ & 50.23 & 43.25 \\
$4-15-1$ & 50.17 & 43.25 \\
$4-16-1$ & 50.22 & 43.35 \\
$4-30-1$ & 50.17 & 43.54 \\
$4-61-1$ & 49.92 & 56.75 \\
$4-99-1$ & 51.19 & 56.75 \\
\hline
\end{tabular}

The learning rate that influences the performance of the system is also being factored in. The rate of learning is between 0.001 to 0.1 . Table 2 outlines the RMSE as well as the percentage of accuracy. Based on Table 3, even though the rate of learning of 0.1 contributes to a better percentage of accuracy (56.74\%), the RMSE value has proven to be higher (63.26\%). The rate of learning of 0.1 gives a lower value of RMSE which is $49.92 \%$. Therefore, the RBFNN with the hidden node of 61 and the rate of learning of 0.01 is used during the training, validation as well as testing processes. The optimal weighted value that has been trained by the system will then be used for the testing process.

The second objective of this study is to analyze the influence of the selected data input variables on the performance of the RBFNN model in the rainfall forecasting. [12] highlights that the selection of suitable data input plays a critical role in reducing the time required to train the RBFNN model while improving the overall accuracy of the results. Figures 2(a)-(c) illustrate the relationship patterns between the cumulative rainfall data with parameters such as temperature, humidity as well as air pressure. Figure 2(a) demonstrates that the cumulative daily rainfall in Malaysia is less than $60 \mathrm{~mm}$ and the rainfall data shows higher concentration between $30^{\circ} \mathrm{C}$ and $33^{\circ} \mathrm{C}$ in temperature. In terms of humidity, as shown in Figure 2(b), the cumulative rainfall data shows a comprehensible pattern when the humidity is between $40 \%$ to $90 \%$ for daily rainfall of less than $60 \mathrm{~mm}$. When illustrating the relationship of cumulative daily rainfall against air pressure, Figure 2 (c) proves that the daily rainfall and air pressure are correlated to each other ranging from 1007 mbar up to 1013 mbar for cumulative daily rainfall less than $40 \mathrm{~mm}$.

Second evaluation of the meteorological data revolves around the assessment of the performance of the data input of the network model. A lower value of RMSE indicates a better rainfall forecast, this has been demonstrated by the data outlined in Table 3 where a lower RMSE was recorded when parameters such as temperature, humidity and air pressure were introduced. The percentage of accuracy also increases by $5 \%$ thus, has further proven the importance of draw the 
Table 2. The radial basis function neural network model performance for learning rate.

\begin{tabular}{ccc}
\hline Learning rate & RMSE & Percentage of accuracy (\%) \\
\hline 0.0001 & 63.26 & 56.74 \\
0.001 & 51.11 & 56.75 \\
0.01 & 49.92 & 56.75 \\
0.1 & 50.21 & 43.25 \\
\hline
\end{tabular}

Table 3. Meteorological data influence on the radial basis function neural network model.

\begin{tabular}{ccc}
\hline Input data & RMSE & Percentage of accuracy (\%) \\
\hline $\begin{array}{c}\text { Rainfall and temperature } \\
\text { Rainfall, temperature, humidity } \\
\text { and air pressure }\end{array}$ & 50.59 & 51.24 \\
\hline
\end{tabular}

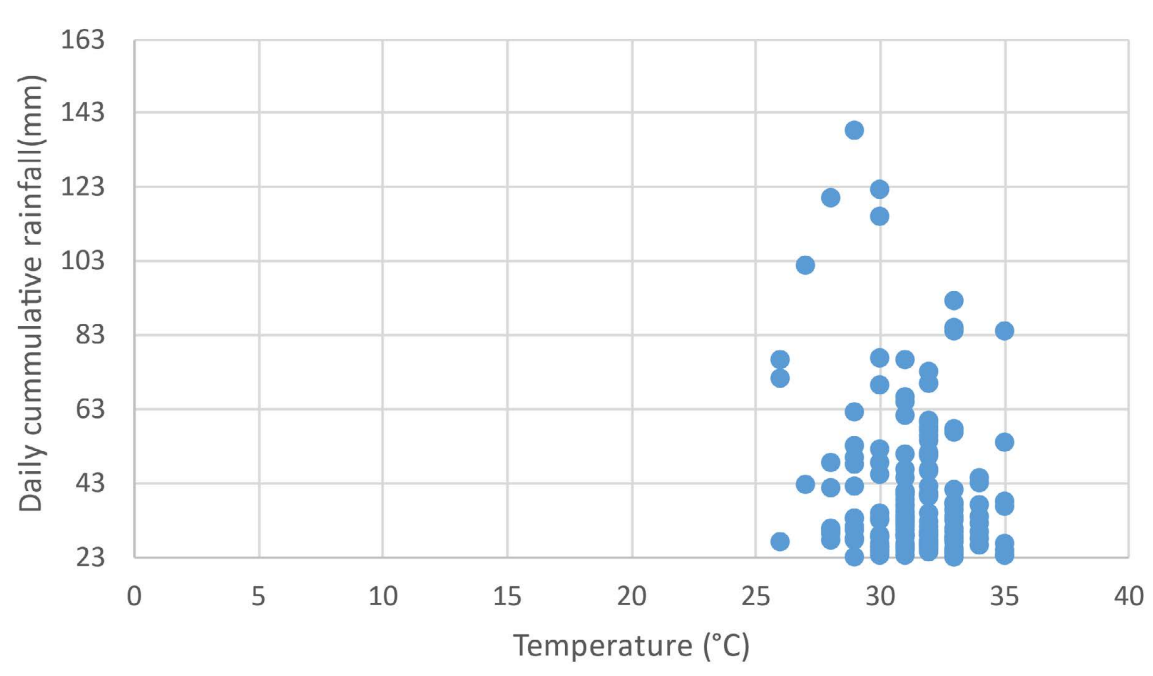

(a)

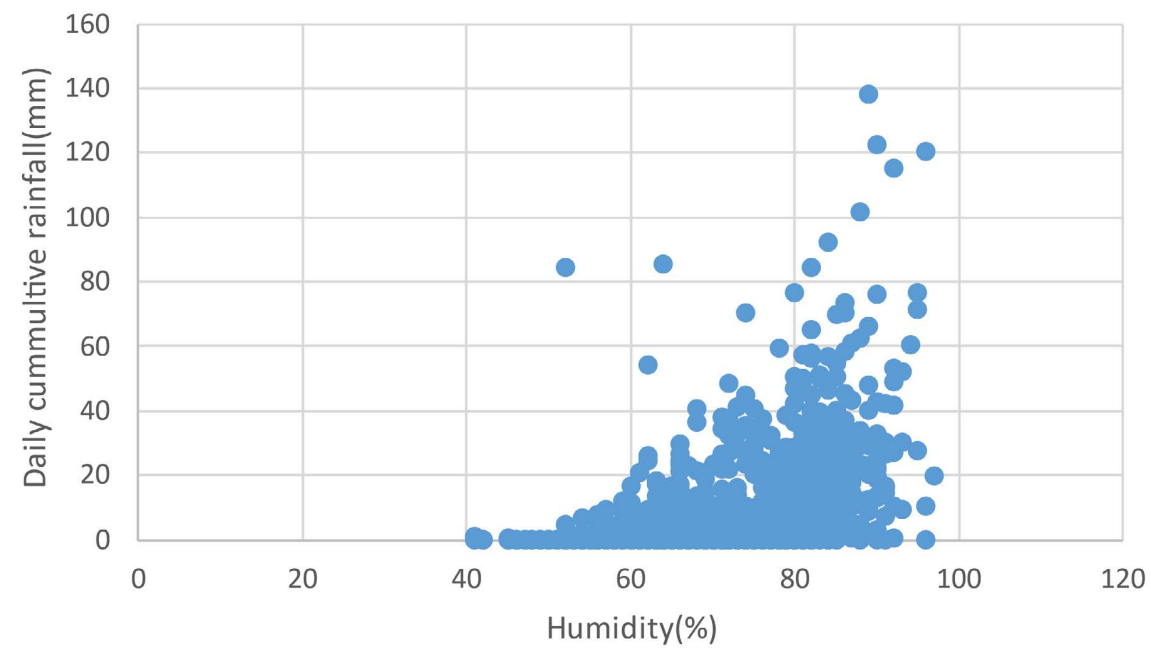

(b) 


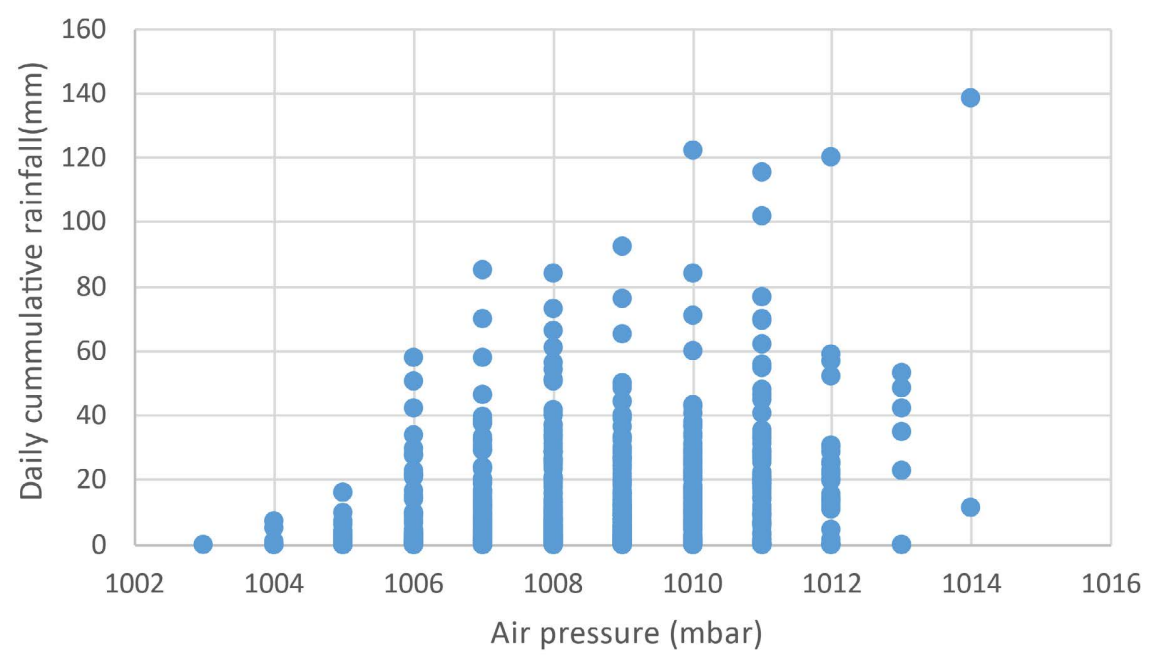

(c)

Figure 2. (a) Relationship pattern between temperature $\left({ }^{\circ} \mathrm{C}\right)$ and daily cummulative rainfall (mm); (b) Relationship Pattern between Humidity (\%) and cumulative daily rainfall $(\mathrm{mm})$; (c) relationship pattern between air pressure (mbar) and cumulative daily rainfall $(\mathrm{mm})$.

relationships between other parameters in the underlying meteorological data with the rainfall forecast. This study also paves way to future studies to explore other meteorological data parameters that could potentially influence the accuracy of rainfall forecasting such as sea level, minimum as well as maximum temperature similar to a study conducted by [12].

\section{Conclusion}

Based on the radial basis function neural network scheme, this paper conducted the performance of weather parameters analysis that affects the performance of rain forecasting system. Based on this study, it has been made explicit that the higher value of hidden nodes results shows a better performance of the rainfall forecasting system, but it will add more complexity to the neural network model and to the training process involved. Plus, the overall outcome outlines that the selection of optimal hyperparameters of the RBFNN model as well as accurate meteorological data had impact on the performance of the RBFNN model. The accuracy and the performance of the system can be enhanced further by adding in more factors in subsequent studies. This study will be useful in the application of tree management system as it incorporates the essential weather element.

\section{Conflicts of Interest}

The authors declare no conflicts of interest regarding the publication of this paper.

\section{References}

[1] Avolio, M.L., Pataki, D.E., Gillespie, T.W., Jenerette, G.D., McCarthy, H.R., Ste- 
pha-nie, P. and Clarke, L.W. (2015) Tree Diversity in Southern California's Urban Forest: The Interacting Roles of Social and Environmental Variables. Frontier in Ecology and Evolution, 3, 3-15. https://doi.org/10.3389/fevo.2015.00073

[2] Mallikarjun, M., Farheen, Saraswathi, L. and Prakash, L. (2020) Artificial Neural Network Based Weather Pridiction System. International Journal of Scientific \& Technology Research, 9, 5587-5594.

[3] Iqbal, H., Rasel, H.M., Monzur, A.I. and Mekanik, F. (2019) Long-Term Seasonal Rainfall Forecasting Using Linear and Non-Linear Modelling Approaches: A Case Study for Western Australia. Meteorology and Atmospheric Physics, 132, 131-141. https://doi.org/10.1007/s00703-019-00679-4

[4] Lee, J., Kim, C.G., Lee, J.E., Kim, N.W. and Kim, H. (2018) Application of Artificial Neural Networks to Rainfall Forecasting in the Geum River Basin, Korea. Water, 10, 1448. https://doi.org/10.3390/w10101448

[5] Ishwarya, G., Santhrupthi, M.B., Shanthi, B. and Varsha, N. (2021) Prediction of Rainfall Using Machine Learning Algorithms. International Journal of Scientific Research \& Engineering Trends, 7, 2124-2128.

[6] Suhaimi, S. and Rosmina, A.B. (2009) Rainfall Runoff Modeling Using Radial Basis Function Neural Network for Sungai Tinjar Catchment, Miri, Sarawak. UNIMAS E-Journal of Civil Engineering, 1, 1-7. https://doi.org/10.33736/jcest.66.2009

[7] Mansor, M.A., Mohd Jamaludin, S.Z., Mohd Kasihmuddin, M.S., Alzaeemi, S.A., Md Basir, M.F. and Sathasivam, S. (2020) Systematic Boolean Satisfiability Programming in Radial Basis Function Neural Network. Processers, 8, 1-16. https://doi.org/10.3390/pr8020214

[8] Cramer, S., Kampouridis, M., Freitas, A.A. and Alexandridis, A.K. (2017) An Extensive Evaluation of Seven Machine Learning Methods for Rainfall Prediction in Weather Derivatives. Expert Systems with Applications, 85, 169-181. https://doi.org/10.1016/j.eswa.2017.05.029

[9] Chai, S.S., Wong, W.K. and Goh, K.L. (2016) Backpropagation vs. Radial Basis Function Neural Model: Rainfall Intensity Classification for Flood Prediction Using Meteorology Data. Journal of Computer Science, 12, 191-200. https://doi.org/10.3844/jcssp.2016.191.200

[10] Vivekanandan, N. (2014) Prediction of Rainfall Using MLP and RBF Networks. International Journal Advanced Networking and Applications, 5, 1974-1979.

[11] Chai, S.S, Wong, W.K, Goh, K.L., Wang, H.H. and Wang, Y.C. (2019) Radial Basis Function (RBF) Neural Network: Effect of Hidden Neuron Number, Training Data Size, and Input Variables on Rainfall Intensity Forecasting. International Journal on Advanced Science Engineering Information Technology, 9, 1921-1926. https://doi.org/10.18517/ijaseit.9.6.10239

[12] Madhiarasan, M. (2020) Accurate Prediction of Different Forecast Horizons Wind Speed Using a Recursive Radial Basis Function Neural Network. Protection and Control of Modern Power Systems, 5, 22. https://doi.org/10.1186/s41601-020-00166-8

[13] Mary, N.A., Thomas, J.A. and Akintunde, A.A. (2017) Rainfall Rate Prediction Based on Artificial Neural Networks for Rain Fade Mitigation over Earth-Satellite link. IEEE Africon 2017 Proceeding, Cape Town, 18-20 September 2017, 579-584.

[14] Broomhead, D.S. (1988) Multivariate Functional Interpolation and Adaptive Networks. Complex Systems, 2, 321-355.

[15] Amrul, F., Hudan, P.A., Shamsul Faisal, M.H., Che Munira, C.R., Aminaton, M. and 
Shahrum, S.A. (2020) Deep Learning-Based Forecast and Warning of Floods in Klang River, Malaysia. International Information and Engineering Technology Association, 25, 365-370. https://doi.org/10.18280/isi.250311 